\title{
Study on the Synergistic Properties of Two Nonionic Natural Gas Hydrate Anti-agglomerants Via Rocking Cell Tests
}

\author{
Sanbao Dong*, Mingzhong Li, Chenwei Liu \\ College of Petroleum Engineering, China University of Petroleum (East China), Qingdao, China \\ Email address: \\ dongsanbao2007@hotmail.com (Sanbao Dong), limingzhong_upc@hotmail.com (Mingzhong Li), liuchenwei1986@126.com (Chenwei Liu) \\ *Corresponding author
}

To cite this article:

Sanbao Dong, Mingzhong Li, Chenwei Liu. Study on the Synergistic Properties of Two Nonionic Natural Gas Hydrate Anti-agglomerants Via Rocking Cell Tests. International Journal of Energy and Power Engineering. Vol. 6, No. 6, 2017, pp. 84-90. doi: 10.11648/j.ijepe.20170606.11

Received: November 7, 2017; Accepted: November 20, 2017; Published: December 6, 2017

\begin{abstract}
The application of anti-agglomerants (AAs) is becoming attractive due to effectiveness at low dosage and high subcooling. However, limited attention has been paid to the synergism effect between different AAs to increase their performance. In this study, anti-agglomeration performance of single and compounded chemical additives using a sapphire rocking cell is evaluated. The experimental results show that cocamidopropyl dimethylamine (AA) combined with sorbitan monooleate (Span 80) exhibits good anti-agglomeration performance. A compounded anti-agglomeration mechanism, in which Span 80 promotes the dispersion of water droplet in the oil phase before the formation of hydrates and AA prevents the agglomeration of hydrate particles formed from water droplets, is proposed. The physical appearance of the octane/brine/AAs mixtures has been studied and related to the anti-agglomeration performance of the AAs.
\end{abstract}

Keywords: Gas Hydrates, Hydrate Anti-agglomeration, Synergistic Effect, Span 80, Salt

\section{Introduction}

Gas hydrates are ice-like crystalline compounds composed of hydrogen bonded water and small gas molecules (e.g., methane, ethane, propane, carbon dioxide and nitrogen) that often form in gas and oil pipeline at high pressure and low temperature conditions [1]. The formation of gas hydrates in the flowlines may lead to the aggregation and deposition of these crystalline particles, which may completely block the flowlines, causing operational and safety hazards [2].

Traditionally, thermodynamic hydrate inhibitors (THIs) are employed to mitigate the risk of hydrate formation in flowlines. However, large amount of these THIs (e.g., up to 60 $\mathrm{wt} \%$ in the aqueous phase) are required, which makes this method cost prohibitive and less attractive [2-3]. Currently, there is an increasing interest in developing low dosage hydrate inhibitors (LDHIs) to control the hydrate plugging risk. LDHIs can be effective in low concentrations ( $<2.0 \mathrm{wt} \%$ based on the aqueous phase) [2,4]. There are two kinds of LDHIs: kinetic hydrate inhibitors (KHIs) and hydrate anti-agglomerants (AAs). KHIs are usually water soluble polymers, which can inhibit nucleation and slow down the growth rate of hydrate crystals. KHIs may be ineffective when subcooling is over $10^{\circ} \mathrm{C}[2,5]$. As gas and oil exploration moves to deeper water ( $>8000 \mathrm{ft}$ of seawater), AAs become an attractive option because of effectiveness at high subcooling or shut-in conditions [6]. AAs are surfactants that allow hydrate to form, but keep the hydrate particles dispersed in hydrocarbon phase.

Typical AAs are quaternary ammonium salts (QAs), which was first explored by Shell in 1993 [2, 7]. QAs have been commercialized and employed in a number of fields $[3,8]$. However, most QAs are considered harmful to the environment because of the high toxicity and limited biodegradation [9]. Greener, more effective and cost-competitive AAs still remain to be further explored. Kelland et al., investigated the AA performance of some zwitterionic surfactants (betaine surfactants), which are greener than the QAs, in synthetic natural gas $(\mathrm{SNG}) /$ condensate/synthetic sea water $(\mathrm{SSW}=3.6 \%)$ system using high pressure sapphire apparatus and wheel simulator apparatus [10]. It was found that $3-[N$, 
$N$-dibutyl- $N$-(2-(3-carboxy-pentadecenoyloxy)-propyl)] ammonia propanoate exhibited the best performance, which could successfully prevent hydrate anti-agglomeration in sapphire cell at a concentration of $0.5 \mathrm{wt} \%$ (based on the aqueous phase), a water cut of $20 \%$, a subcooling of $15.9^{\circ} \mathrm{C}$ and a pressure of $122 \mathrm{bar}$. However, the performances of the best zwitterionic surfactants were not as well as the commercial QAs. On the other hand, a number of nonionic surfactants, which are thought to be non-toxic, have been investigated as AAs in laboratory researches. Sloan and coworkers found that a series of Sorbitan surfactants can function successfully as AAs at low water cuts, although a dosage of $3 \mathrm{wt} \%$ is required to realize anti-agglomeration [11]. They also found that some nonionic surfactants containing caprolactam and alkylamide groups (e.g. dodecyl-2-(2-caprolactamyl) ethanamide) can provide good performance in anti-agglomeration. However, the preparation of these synthetic surfactants may be costly. Kelland et al., investigated the performance of a series of alkylamide surfactants AAs and found that some of these surfactants could be used as AAs, but their performance were not as good as commercial QAs [12]. Rhamnolipid surfactants, which are biodegradable and less toxic surfactants, have been shown to be effective in anti-agglomeration in model oil, water and tetrahydrofuran system. Alcohol cosurfactants may increase the performance of Rhamnolipid surfactants [13]. Biosurfactants extracted from plants have also been proved to be effective in anti-agglomeration as AAs. Wang et al. [14] found that the active components, which were extracted from a terrestrial plant fruit, provide good performance in anti-agglomeration in natural gas, diesel and water system at $1{ }^{\circ} \mathrm{C}, 20 \%$ water cut and a dosage of $0.4 \mathrm{wt} \%$ based on the aqueous phase. Their results indicated that main active components were found to be apigenin and luteolin. The best results were obtained if the plant-extract $\mathrm{AA}$, which contained mixtures of all the extracted components, were employed as the AAs in the test. However, the active components of the plant-extracted AAs remain to be further separated and identified. Sun and Firoozabadi [15] found a new nonionic surfactant, cocamidopropyl dimethylamine (AA), could work in pure water system. However, a recent study showed that the effective dosage of AA decreased when the water cut was decreased to $10 \%$ in octane/methane/brine system [16].

Synergistic anti-agglomeration could be an effective means to enhance the performance of AAs and to reduce the effective dosages of AAs. However, limited studies on the performance of compounded nonionic surfactants have been carried out. Synergism in the application of hydrate anti-agglomeration needs to be further explored. Yan et al. found that the performance of a plant-extracted AAs could be increased when used in combination with Span 20. The active component of the plant-extracted AAs still remains unknown [17]. Chen et al. proved that AEO-3 combined with sorbitan surfactants (e.g. Span 20) exhibits higher performance in hydrate anti-agglomeration at low water cuts (up to $30 \%$ ) in water, diesel oil and natural gas system. However, a concentration of $3 \mathrm{wt} \%$ was required to achieve anti-agglomeration [18].

The purpose of this work is to investigate the synergistic effect between AA and Span 80 on methane hydrate anti-agglomeration at high pressure (100 bar) and water cuts of 10,20 and $30 \%$ by mixing n-octane and the aqueous phase (fresh water or brine). N-octane and pure methane are employed as the hydrocarbon and gas phase, respectively. Performance testing is conducted in high pressure rocking cell apparatus, which has been used to investigate the performance of AAs in several works $[11,13]$. The physical appearance (phase behavior) of the emulsions stabilized by AA and Span 80 is also measured to interpret the anti-agglomeration performance of AA and Span 80 .

\section{Materials and Methods}

\subsection{Materials}

The AA used in this study is provided by Lubrizol Corporation. It consists of $80-90 \%$ cocamidopropyl dimethylamine (AA) in solvent consisting of glycerin (at $5-10 \%$ ), small amount of free amine and water. Span 80 (> 99\% Sorbitan Monooleate) is from Tokyo Chemical Industry Ltd. (TCI). Figure 1 shows the chemical structure of AA and Span 80 . Sodium chloride (99.8\%) is purchased from Fisher. Octane (97\%, Acros Organics) and Methane gas $(99.99 \%$ ultrahigh purity, MADCO) are used as the liquid hydrocarbon and gas hydrate former, respectively.

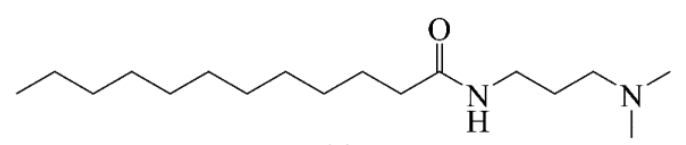

(a)

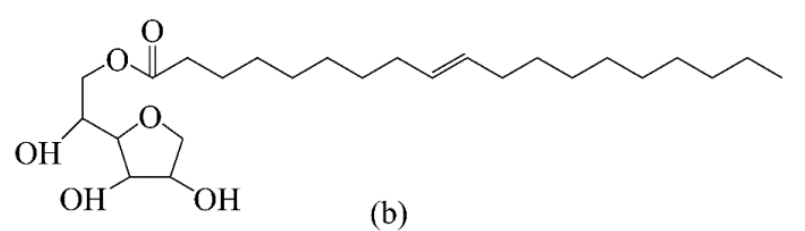

Figure 1. Chemical structures of $A A(a)$ and Span 80 (b).

\subsection{AA Performance}

The AAs performance tests are conducted in a high pressure sapphire rocking cell equipment (PSL RCS-2) shown in Figure 2. Each cell has a volume of $20 \mathrm{~mL}$ equipped with a stainless steel ball, which runs back and forth and provides agitation of fluids during rocking. The inner diameter of the cell is $0.5 \mathrm{in}$. $(1.27 \mathrm{~cm})$ and the diameter of the stainless steel ball is $0.4 \mathrm{in}$. $(1.02 \mathrm{~cm})$. Each cell is fitted with two position sensors to record the ball running time and a pressure sensor to record the pressure in the cell. In each test, the cells are filled with $10 \mathrm{~mL}$ liquid mixtures containing surfactants, octane and aqueous phase. The cells are placed into a water bath which is connected to an external chiller. After the water bath is filled with water, the cells are flushed with pure methane three times to remove the residual air in the cells and pipelines and then the cells are pressurized with methane to a pressure of around 100 bar. After 
the cells are charged with various samples, they are rocked at the room temperature for $30 \mathrm{~min}$ to reach equilibrium. Then the water bath is cooled from room temperature to $2^{\circ} \mathrm{C}$ at a cooling rate of $4^{\circ} \mathrm{C} / \mathrm{h}$ while the cells are being rocked. The cells are then kept at $2{ }^{\circ} \mathrm{C}$ for 4 hours allowing the hydrate to fully develop before the temperature ramps back to the initial temperature. During the cooling and hydrate formation, additional methane is intermittently charged into the cells to maintain a constant pressure of 100 bar. In each test, the cells are rocked with a frequency of 15 times per minute at an angle of $\sim 45^{\circ}$. The ball running time, cell pressure and bath temperature are recorded during each test.

\subsection{Emulsion Formation and Phase Behaviors}

The state of mixture with varying water cuts (10 to 30\%) and salinities ( $0 \mathrm{wt} \% \mathrm{NaCl}$ and $4 \mathrm{wt} \% \mathrm{NaCl}$ ) are firstly visually examined. This part of the experiments are performed at ambient temperature and pressure with surfactants, octane and the aqueous phase in 15-mL centrifuge tubes purchased from Fisher Scientific. The total liquid volume at atmospheric condition is $10 \mathrm{~mL}$. These data correspond to the conditions at atmospheric pressure when there is very little solubility of methane in the octane. The mixture of surfactants, octane and the aqueous phase is thoroughly homogenized by hand shaking for 10 minutes and then kept undisturbed. The phase distribution is recorded at 1 minute, 10 minutes and one hour after mixing. The physical appearances of the mixtures are observed and recorded.

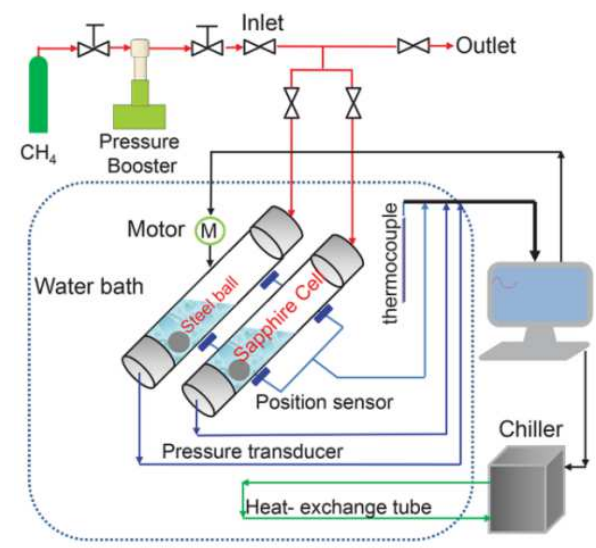

Figure 2. Schematic of the Rocking Cell (RCS-2).

\section{Results and discussion}

\subsection{Performance of $A A$}

The performance of AA at 10 to $30 \%$ water cut are firstly tested. As shown in Table $1,1 \mathrm{wt} \% \mathrm{AA}$ is not sufficient to prevent the hydrate aggregation at $10 \%$ water cut. Hydrate blockage occurs because of the agglomeration of hydrate particles, as seen in Figure 3 (a), in which the steel ball is trapped in the hydrates located at the top of the cell. The minimum effective dosage of AA in fresh water turns out to be $1.4 \mathrm{wt} \%$. However, with the presence of $4 \mathrm{wt} \% \mathrm{NaCl}$ in the aqueous phase, $1.6 \mathrm{wt} \% \mathrm{AA}$ is required to achieve anti-agglomeration. $\mathrm{NaCl}$ lowers the performance of the $\mathrm{AA}$, which may due to the decreased AA concentration in the aqueous phase caused by the strong interactions between salt ions and water molecules. At $20 \%$ water cut, the effective dosages of AA in fresh and brine water are found to be $1.4 \mathrm{wt} \%$ and $0.8 \mathrm{wt} \%$, respectively. $\mathrm{NaCl}$ increases the performance of $\mathrm{AA}$ at this condition. At $30 \%$ water cut, $1 \mathrm{wt} \%$ AA can successfully prevent the hydrate agglomeration when there is no salt in the aqueous phase. When $4 \mathrm{wt} \% \mathrm{NaCl}$ is added into the aqueous phase, $0.8 \mathrm{wt} \% \mathrm{AA}$ is found to be sufficient to realize hydrate anti-agglomeration. It can be found that the minimum effective dosages of AA increases as the water cut increased. The reason may be due to the decreased stability of the emulsion at low water cut, which will be further discussed later. In systems with lower water cuts, higher concentrations of AA are required to prevent hydrate blockage, which inspires us to use a co-surfactant, Span 80 , to reduce the concentration of AAs.

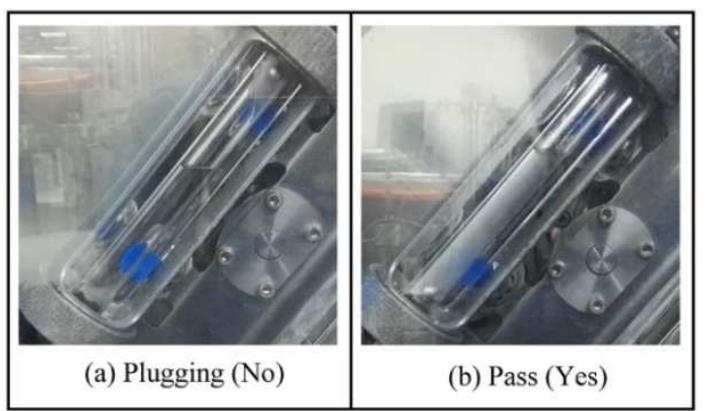

(a) Pass (No) 1 wt\% AA, and (b) Pass (Yes) 1.4 wt $\%$ AA.

Figure 3. Visual observations of AA effectiveness: Water cut is $10 \%$.

Table 1. Effectiveness of AA in methane/octane/aqueous phase at 10, 20 and $30 \%$ water cuts.

\begin{tabular}{llllll}
\hline Aqueous $(\mathbf{m L})$ & Octane $(\mathbf{m L})$ & Water cut $\mathbf{( \% )}$ & $\mathbf{N a C l} \mathbf{( w t} \%)$ & $\mathbf{A A}(\mathbf{w t} \%)$ & Effectiveness \\
\hline 1 & 9 & 10 & 0 & 1 & No \\
1 & 9 & 10 & 10 & 1 & No \\
1 & 9 & 10 & 0 & 1.4 & Yes \\
1 & 9 & 10 & 4 & 1.6 & Yes \\
2 & 8 & 20 & 0 & 1.4 & No \\
2 & 8 & 20 & 4 & 0.6 & Yes \\
2 & 8 & 20 & 4 & 0.8 & No \\
2 & 8 & 20 & 0 & 0.8 & Yes \\
3 & 7 & 30 & 0 & 1 & No \\
3 & 7 & 30 & 4 & 0.6 & Yes \\
3 & 7 & 30 & 4 & 0.8 & No \\
3 & 7 & 30 & & Yes \\
\hline
\end{tabular}




\subsection{Performance of Compounded Anti-agglomerants}

In order to reduce the usage of the AA/AAs at low water cuts (e.g. $<30 \%$ ), Span 80 is used together with AA to see if there is synergy. Span 80 has a hydrophilic-lipophilic balance (HLB) value of 4.3 , which is considered as an oil soluble surfactant that can promote the formation of water-in-oil emulsion. Span 80 has been used as a hydrate anti-agglomerant in labroratory researches $[19,20]$. In this study, the synergistic effect between AA and Span 80 is observed. As listed in Table 2, at 10\% water cut without salt, the effective dosage of Span 80 for anti-agglomeration is $2 \mathrm{wt} \%$. However, when $4 \mathrm{wt} \% \mathrm{NaCl}$ is introduced into the aqueous phase, $3 \mathrm{wt} \% \mathrm{Span} 80$ is required to realize hydrate anti-agglomeraiton. $\mathrm{NaCl}$ reduces the performance of Span 80 , which may due to the reduced concentration of Span 80 in the bulk water phase caused by the strong ion strength. The strong interactions between salt ions and water molecules may leads to the migration of Span 80 molecules from the aqueous phase into the oil phase and the phase separation of emulsions which will be discussed later when we present emulsion measurement. The synergy tests are conducted by varying the concentration ratios of AA and Span 80 at different total concentrations, such as $0.4,0.6,0.8$ and 1.0 wt $\%$. At $10 \%$ water cut, we found that $1 \mathrm{wt} \%$ concentration of the mixtures of AA and Span 80 can lead to hydrate anti-agglomeration. In systems without salt, a concentration ratio ranging from 1:2 to $1: 3$ of AA to Span 80 is sufficient to prevent hydrate blockage. With the presence of $4 \mathrm{wt} \% \mathrm{NaCl}$ in the aqueous phase and a total concentration of $1 \mathrm{wt} \% \mathrm{AA}$ and Span 80 , a ratio ranging from 2:1 to 1:4 of AA to Span 80 can realize hydrate anti-agglomeration.

Table 2. Synergistic effect of Span 80 and AA at different ratios (weight basis). Liquid (brine/octane) volume in Sapphire cell is $10 \mathrm{~mL}: 10 \% \mathrm{water}$ cut.

\begin{tabular}{|c|c|c|c|c|c|c|}
\hline Aqueous (mL) & Octane $(\mathrm{mL})$ & AA (wt\%) & Span $80(w t \%)$ & AA/Span 80 ratio & $\mathrm{NaCl}(\mathrm{wt} \%)$ & Effectiveness \\
\hline 9 & 1 & 1.4 & 0 & & 0 & Yes \\
\hline 9 & 1 & 1 & 0 & & 0 & No \\
\hline 9 & 1 & 0.8 & 0.2 & $4 / 1$ & 0 & No \\
\hline 9 & 1 & 0.75 & 0.25 & $3 / 1$ & 0 & No \\
\hline 9 & 1 & 0.67 & 0.33 & $2 / 1$ & 0 & No \\
\hline 9 & 1 & 0.5 & 0.5 & $1 / 1$ & 0 & No \\
\hline 9 & 1 & 0.33 & 0.67 & $1 / 2$ & 0 & Yes \\
\hline 9 & 1 & 0.25 & 0.75 & $1 / 3$ & 0 & Yes \\
\hline 9 & 1 & 0.2 & 0.8 & $1 / 4$ & 0 & No \\
\hline 9 & 1 & 0 & 1 & & 0 & No \\
\hline 9 & 1 & 0 & 2 & & 0 & Yes \\
\hline 9 & 1 & 1.6 & 0 & & 4 & Yes \\
\hline 9 & 1 & 1.4 & 0 & & 4 & No \\
\hline 9 & 1 & 1 & 0 & & 4 & No \\
\hline 9 & 1 & 0.8 & 0.2 & $4 / 1$ & 4 & No \\
\hline 9 & 1 & 0.75 & 0.25 & $3 / 1$ & 4 & No \\
\hline 9 & 1 & 0.67 & 0.33 & $2 / 1$ & 4 & Yes \\
\hline 9 & 1 & 0.5 & 0.5 & $1 / 1$ & 4 & Yes \\
\hline 9 & 1 & 0.33 & 0.67 & $1 / 2$ & 4 & Yes \\
\hline 9 & 1 & 0.25 & 0.75 & $1 / 3$ & 4 & Yes \\
\hline 9 & 1 & 0.2 & 0.8 & $1 / 4$ & 4 & Yes \\
\hline 9 & 1 & 0 & 1 & & 4 & No \\
\hline 9 & 1 & 0 & 2 & & 4 & No \\
\hline 9 & 1 & 0 & 3 & & 4 & Yes \\
\hline
\end{tabular}

At $20 \%$ water cut, the synergistic effect between AA and Span 80 is also observed (table not shown). Results show that without salt, at least $3 \mathrm{wt} \%$ Span 80 is required to realize hydrate anti-agglomeration. However, $5 \mathrm{wt} \%$ Span 80 is necessary for anti-agglomeration in the presence of $4 \mathrm{wt} \% \mathrm{NaCl}$ in the aqueous phase. The synergistic effect of AA and Span 80 is tested by varying the concentrations of AA and Span 80 at a total concentration of $1 \mathrm{wt} \%$. It is found that $1 \mathrm{wt} \%(\mathrm{AA}+\mathrm{Span}$ 80 ) is sufficient to prevent hydrate blockage. A concentration ratio of AA to Span 80 from 1:1 to 1:3 is optimal for hydrate anti-agglomeration with the absence of salt. When $4 \mathrm{wt} \% \mathrm{NaCl}$ is introduced into the aqueous phase, the minimum dosage of the mixture of AA and Span 80 is found to be $0.6 \mathrm{wt} \%$. A concentration ratio ranging from 4:1 to 1:3 of AA to Span 80 is optimal for hydrate anti-agglomeration.

At $30 \%$ water cut, pure Span 80 is found to be not effective at concentration up to $6 \mathrm{wt} \%$ (table not shown). When Span 80 is used in combination with AA in fresh water, the minimum effective concentration of (AA + Span 80 ) is $1 \mathrm{wt} \%$, which is equal to the minimum effective dosage of pure $\mathrm{AA}$ at the same conditions. There is no appreciable synergy effect of the surfactants. However, with the presence of $4 \mathrm{wt} \% \mathrm{NaCl}$ in the aqueous phase, $0.6 \mathrm{wt} \%$ of the mixtures of AA and Span 80 is enough to prevent hydrate blockage. A concentration ratio range of 4:1 to 1:2 of AA to Span is optimal for hydrate anti-agglomeration. From the evaluation of the synergistic effect of the AA and Span 80, it is found that the synergistic effect for Span 80 is much weaker at $30 \%$ water cut than at 10 and $20 \%$ water cut. The mechanism of the synergistic effect of AA and Span 80 will be discussed later.

\subsection{Emulsion Phase Behavior}

The emulsion phase behavior is tested within centrifugal 
tubes, each of the vials consists the mixtures of brine/water/octane/surfactants at a total volume of $10 \mathrm{~mL}$ and a total concentration of the mixtures of AA and Span 80 of 1 $w t \%$ (based on the aqueous phase). Figure 4 shows the phase behaviors of the mixtures at $10 \%$ water cut stabilized by AA and Span 80. The ratio of AA and Span 80 is labelled on the tubes. As shown in Figure 4, in each tube without salt, phase separation occurs within minutes after the mixing. For the samples with $1 \mathrm{wt} \%$ AA, mixtures separate into three layers, which are the octane phase in the upper layer, a free water phase in the bottom layer and a thin emulsion phase in the middle layer. However, for each sample contains $1 \mathrm{wt} \%$ Span 80 or $1 \mathrm{wt} \%$ (AA + Span 80$)$, only two layers after the mixing are observed, which are octane phase in the upper layer and the water-in-oil emulsion phase in the bottom layer. In Figure 4 , the emulsion with $1 \mathrm{wt} \%$ AA exhibits the lowest stability, as indicated by the appearance of bulk water phase and the smallest amount of emulsion. In each tube contains the mixtures of AA and Span 80, the emulsion phase exhibits higher stability. Bulk water phase is not observed within 10

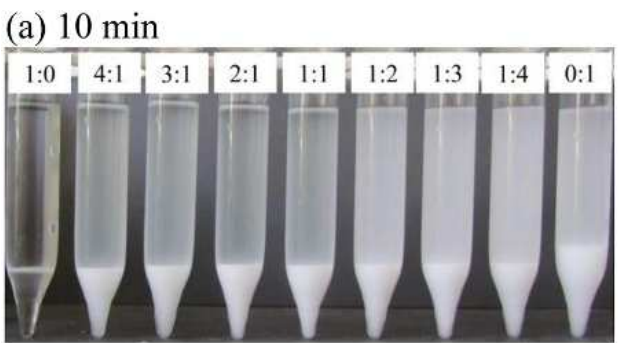

minutes, which may due to the increased emulsion stability by Span 80 . In the sample containing $1 \mathrm{wt} \%$ Span 80 , the amount of emulsion phase (water-in-oil emulsion) is much higher than that of other samples, revealing the superior performance of dispersing water droplets into octane phase. We also find that when $4 \mathrm{wt} \% \mathrm{NaCl}$ is introduced into the aqueous phase, the amount of the emulsion phase is significantly reduced, indicating that the emulsion stability of Span 80 is decreased by salt. This may be the reason that 2 wt $\%$ Span 80 is sufficient in fresh water but ineffective in brine water (4 wt \% $\mathrm{NaCl})$.

At 20 and $30 \%$ water cuts, the phase behaviors are similar with that of the mixtures at $10 \%$ water cut (figures not shown). We can find that for samples with $1 \mathrm{wt} \% \mathrm{AA}$, as the water cut increases, the stability of the emulsion has increased. This may be the reason that, in brine $(4 \mathrm{wt} \% \mathrm{NaCl}), 0.8 \mathrm{wt} \% \mathrm{AA}$ is effective at 20 and $30 \%$ water cut, but ineffective at $10 \%$ water cut. This may also be the reason that, in fresh water, $0.8 \mathrm{wt} \%$ AA is effective but ineffective at 10 or $20 \%$ water cut.

\section{(b) $8 \mathrm{hr}$}

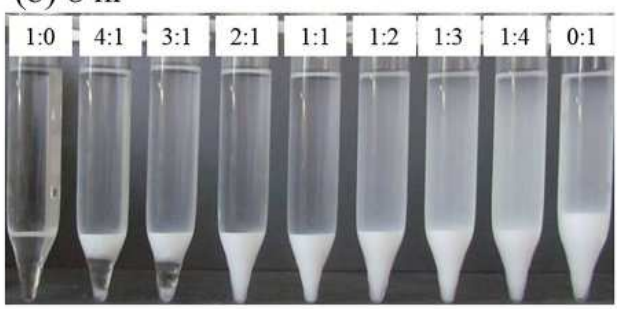

No salt

(c) $10 \mathrm{~min}$

(d) $8 \mathrm{hr}$
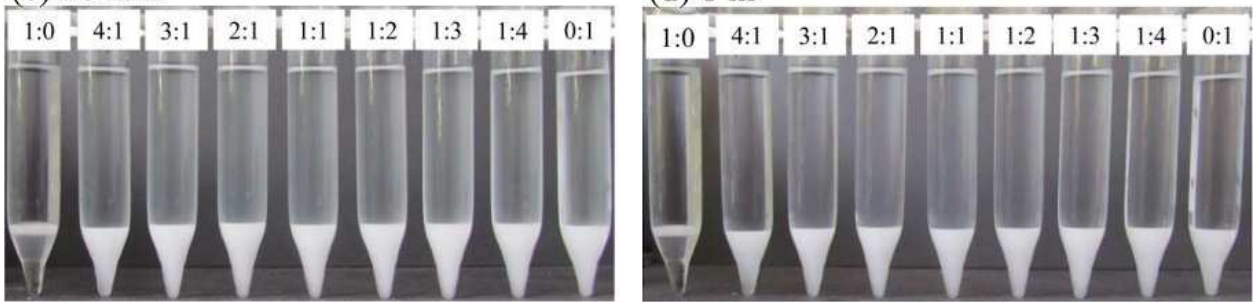

$4 \mathrm{wt} \% \mathrm{NaCl}$

Figure 4. Phase and emulsion distributions of mixtures with AA and Span 80 and water cut of 10\% at time t=10 min (a, c) and $t=8$ hr (b, d) after mixing. Each sample contains $1 \mathrm{wt} \%(\mathrm{AA}+\mathrm{Span} 80)$ and $0 \mathrm{wt} \% \mathrm{NaCl}$ (top, a and b) and $4 \mathrm{wt} \% \mathrm{NaCl}$ (bottom, c and d). The mass ratio of AA to Span 80 is shown on the top of each tube.

\subsection{Proposed Mechanism}

As an oil soluble surfactant, Span 80 would increase the stability of the water-in-oil emulsion and facilitate the dispersion of hydrate particles which are converted from water droplets. Based on the oil-soluble nature of Span 80, we suggest the following mechanism of synergistic effect of AA and Span 80 in realizing hydrate anti-agglomeration, as shown in Figure 5. The mixtures of AAs/brine/oil (Figure 5(a)) are emulsified because of the homogenization. Water bulk water phase is dispersed into water droplets suspending in the oil phase (Figure 5(b)). Before hydrate formation, the dispersion of water droplets into oil phase is promoted by Span 80 and AA, which have been adsorbed onto the water/oil interfaces. Once the hydrate formation starts, another component, the AA, plays a main role in inhibiting the aggregation of the hydrate particles formed from water droplets (Figure 5(c)). Most of the molecules adsorbed onto the interfaces of hydrate/oil are considered to be the AA molecules. The AA molecules are considered to have the advantage of stronger adsorption onto the surface of hydrate particles than Span 80 , because the hydrogen bond between AA and hydrate surface is stronger than the hydrogen bond between Span 80 and hydrate surface. This explanation is based on the enthalpy of hydrogen bond N-H-O (from AA) and O-H-O (from Span 80), which are 29 and $21 \mathrm{KJ} \cdot \mathrm{mol}^{-1}$, respectively [21]. With the combined function of the dual surfactant system, the water-in-oil emulsion could be more stable and the hydrate anti-agglomeration will possibly be enhanced. Synergy effect 
has also been observed by Chen et al. [18] when they test the performance of the mixture of AEO-3 and Span 20 as hydrate anti-agglomerants in (diesel + water + natural gas) system. At $30 \%$ water cut with the addition of $3 \mathrm{wt} \%$ (AEO-3 + Span 20),

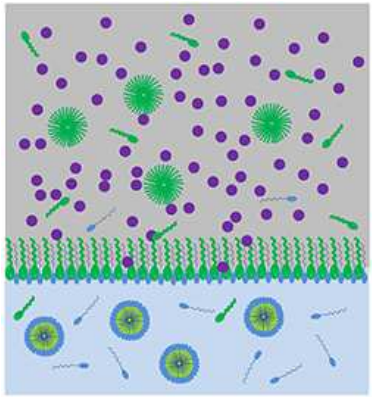

(a) Water/oil/surfactants/gas system before emulsification

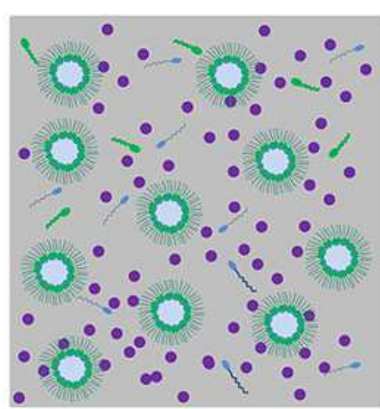

(b) Water dispersed into oil phase by surfactants

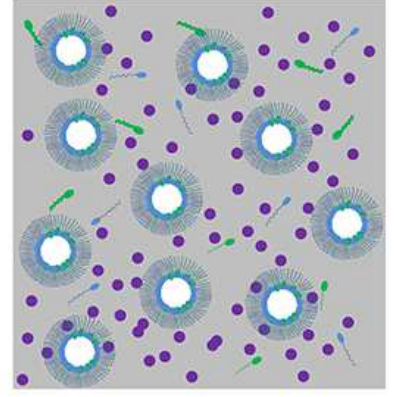

(c) Hydrate formation and dispersion in oil phase

$\begin{array}{llll}- \text { AA } & \text { Span } 80 & \text { Methane } & \\ \text { (c) AA micelle } & \text { Oil phase }\end{array}$

Figure 5. Compounded working mechanism of AA and Span 80 in hydrate anti-agglomeration.

\section{Conclusion}

In this paper, the performance of single AAs (AA and Span 80 ) at water cut ranging from 10 to $30 \%$ through rocking cell tests has been investigated. The effect of salt and water cut on the performance of AAs have been discussed. At 10\% water cut, salt decreases the performance of AA, which may because of the decreased concentration of AA in the bulk water phase caused by the strong interactions between salt ions and water droplets. At 20 to $30 \%$ water cut, salt increases the performance of $\mathrm{AA}$. $\mathrm{NaCl}$ increases the stability of the water-in-oil emulsion and inhibits the conversion of water to hydrate. Salt may also promote the adsorption of AA on the hydrate surface. Salt decreases the performance of Span 80 . The synergistic effect of AA and Span 80 has been observed. A mechanism regarding the synergy, in which Span 80 disperses water into oil phase and AA prevents the aggregation of the hydrate particles formed from water droplets, has been proposed.

\section{Acknowledgements}

The authors highly appreciate the great assistance from Professor Abbas Firoozabadi and his Reservoir Engineering Research Institute (RERI) and on experimental apparatus. The authors also acknowledge the support from "the Fundamental Research Funds for the Central Universities" (Grant No. 16CX06028A).

\section{References}

[1] E. D. Sloan, C. A. Koh, Clathrate Hydrate of Natural Gases; CRC Press: Boca Raton, FL, 2008.

[2] M. A. Kelland, History of the development of low dosage hydrate inhibitors, Energy \& Fuels, 2006, 20(3), 825-847.
[3] M. A. Kelland, Production Chemicals for the Oil and Gas Industry; CRC Press: Boca Raton, FL, 2009.

[4] S. Mokhatab, R. J. Wilkens, K. J. Leontaritis, A review of Strategies for solving gas-hydrate problems in subsea pipelines, Energy Sources, Part A: Recovery, Utilization, and Environmental Effects, 2007, 29(1), 39-45.

[5] M. Sun, A. Firoozabadi, G. Chen, C. Sun, Hydrate size measurements in anti-agglomeration at high watercut by new chemical formation, Energy Fuels, 2015, 29(5), 2901-2905.

[6] A. Perrin, O. M. Musa, J. W. Steed, The chemistry of low dosage clathrate hydrate, Chemical Society Review, 2013, 42, 1996-2015.

[7] J. Jeffrey, Inclusion Compounds, vol. 1. Academic Press, 1984, 159-190.

[8] F. M. Mohamed, M. A. Kelland, Tris(tert-heptyl)-N-alkyl-1-ammonium bromides-powerful THF hydrate crystal growth inhibitors and their synergism with poly-vinylcaprolactam kinetic gas hydrate inhibitor, Chemical Engineering Science, 2016, 144, 275-282.

[9] U. C. Klomp, R. Reijnhart, Method for inhibiting the plugging of conduits by gas hydrates, US5879561, 1999.

[10] M. A. Kelland, T. M. Svartaas, J. Øvsthus, T. Tomita, J. Chosa, Studies on some zwitterionic surfactant gas hydrate anti-agglomerants, Chemical Engineering Science, 2006, 61, 4048-4059.

[11] Z. Huo, E. Freer, M. Lamar, B. Sannigrahi, D. M. Knauss, E. D. Sloan, Hydrate plug prevention by anti-agglomeration, Chemical Engineering Science, 2001, 56, 4979-4991.

[12] M. A. Kelland, T. M. Svartaas, J. Øvsthus, T. Tomita, K. Mizuta, Studies on some alkylamide surfactant gas hydrate anti-agglomerants, Chemical Engineering Science, 2006, 61, 4290-4298.

[13] J. D. York, A. Firoozabadi, Alcohol cosurfactants in hydrate antiagglomeration, Journal of Physical Chemistry B, 2008, 112, 10445-10465. 
[14] X. Wang, H. Qin, Q. Ma, Z. Sun, K. Yan, Z. Song, K. Guo, D. Liu, G. Chen, C. Sun, Hydrate antiagglomeration performance for the active components extracted from a terrestrial plant fruit, Energy Fuels, 2017, 31(1), 287-298.

[15] M. Sun, A. Firoozabadi, New surfactant for hydrate anti-agglomeration in hydrocarbon flowlines and seabed oil capture, Journal of Colloid and Interfacial Science, 2013, 402, 312-319.

[16] S. Dong, A. Firoozabadi, Hydrate anti-agglomeration and synergy effect in normal octane at varying water cuts and salt concentrations, The Journal of Chemical Thermodynamics, 2017 (accepted).

[17] K. Yan, C. Sun, J. Chen, L. Chen, D. Shen, B. Liu, M. Jia, M. Niu, Y. Lv, N. Li, Z. Song, S, Niu, G. Chen, Flow characteristics and rheological properties of natural gas hydrate slurry in the presence of AA in a flow loop apparatus, Chemical Engineering Science, 2014, 106, 99-108.
[18] J. Chen, C. Sun, B. Peng, B. Liu, S. Si, M. Jia, L. Mu, K. Yan, G Chen, Screening and compounding of gas hydrate anti-agglomerants from commercial additives through morphology observation, Energy Fuels, 2013, 27(5), 2488-2496.

[19] R. Ambekar, Equilibrium conditions of hydrate-forming pickering emulsions (M. S. thesis), City University of New York, New York, NY, 2012.

[20] C. Liu, M. Li, G. Zhang, C. A. Koh, Direct measurements of the interactions between clathrate hydrate particles and water droplets, Physical Chemistry Chemical Physics, 2015, 17, 20021-20029.

[21] J. Emsley, Very strong hydrogen bonding, Chemical Society Reviews, 1980, 9, 91-124. 